\title{
FPGA based demodulation of laser induced fluorescence in plasmas
}

\author{
Sean W. Mattingly ${ }^{1}$ and Fred Skiff ${ }^{1}$ \\ Department of Physics and Astronomy, University of Iowa, Iowa City, IA 52242 USA
}

(Dated: 15 August 2018)

\begin{abstract}
We present a field programmable gate array (FPGA) based system that counts photons from laser induced fluorescence (LIF) on a laboratory plasma. This is accomplished with FPGA based up / down counters that demodulate the data, giving a background-subtracted LIF signal stream that is updated with a new point as each laser amplitude modulation cycle completes. We demonstrate using the FPGA to modulate a laser at $1 \mathrm{MHz}$ and demodulate the resulting LIF data stream. This data stream is used to calculate an LIF - based measurement sampled at $1 \mathrm{MHz}$ of a plasma ion fluctuation spectrum.
\end{abstract}

Keywords: laser induced fluorescence, plasma, FPGA, fluctuation, correlation

\section{INTRODUCTION}

Laser induced fluorescence (LIF) is a spectroscopic diagnostic on various types of plasmas including small laboratory plasmas upon which it was pioneered ${ }^{1}$, fusion tokamak divertors ${ }^{2}$, etching plasmas ${ }^{3}$, and more exotic plasmas $^{4,5}$. While LIF is primarily used as a diagnostic to find low order moments $\left(n,\langle v\rangle, T_{i}\right)$ of the plasma ion velocity distribution function, it is also used for examining fundamental plasma physics through its ability to nonperturbatively probe the ion distribution function. Fundamental plasma measurements using LIF include local field measurements through optical tagging techniques ${ }^{6}$; motion of the plasma dielectric ${ }^{7}$; and, more recently, kinetic modes and fluctuations derived from correlation functions ${ }^{8}$. These more complicated plasma measurements require not only measurements of the plasma distribution function, but also background subtracted measurements as a function of time. This is especially the case with plasma fluctuation measurements.

Two major drawbacks plague LIF. First, the signal is limited due to Poisson counting statistics. Second, background collision induced fluorescence threatens to flood the LIF signal. A variety of noise reduction techniques circumvent these issues. We focus on a technique to remove background collision induced fluorescence that has been used since the first demonstration of LIF: amplitude modulation of the stimulating laser and demodulation of the resultant modulated LIF signal. Subtracting the demodulated components removes the background collision induced fluorescence.

Traditionally, this background subtraction is achieved with a lock-in amplifier tuned to the modulation frequency of the laser. However, if one wants to measure a laser induced fluorescence signal as a function of time, rather than its strength as a function of wavelength, a lock-in is not useful and instead digitization is warranted. Typically, digitization is accomplished by constant fraction discriminators that convert the photon multiplier tube (PMT) pulses into digital logic pulses. These digitized pulses are then histogrammed and saved for later processing. Or, if discriminators are not available, then raw voltages from the PMTs themselves are sampled and saved to be processed later. Both the digital and analog methods require great cost in storage, acquisition time, and post processing time. Moreover, the problem of removing the background collision induced fluorescence still remains in both cases.

However, there is a demodulation process that may be done at the time of measurement for digitized photon pulses. This technique ${ }^{10}$ uses up / down counters in a digital logic circuit in order to remove the background fluorescence at the time of measurement. It accomplishes this by counting up while the laser is on and counting down while the laser is off. At the end of each laser modulation cycle, it outputs the result. Thus for each laser modulation cycle it obtains the background - subtracted demodulated LIF photon count.

Since this technique is done entirely on digitized PMT signals, field programmable gate arrays (FPGAs) are well suited to implement these up / down counters for five reasons. First, counters do not take up many logic elements on a typical FPGA, leaving ample room for more channels or in place digital signal processing. Second, the hardware development languages for FPGAs support object instantiation so that numerous readout channels can be created easily and in parallel. This is in contrast with the need, for example, to solder and test 32 separate identical up / down circuits for a set of two 16 element PMTs. Instead, this is all accomplished on a single chip. Third, FPGAs are easily reconfigurable through just software and a cable - changes can be made on the fly, without soldering or buying components. Fourth, FPGAs are clocked to an oscillator which is distributed throughout the circuit, easily achieving synchronization between the laser and the up / down counters. Finally, while examples abound of FPGAs seeing more use in larger plasma experiments ${ }^{11-13}$, they are quickly becoming cost effective enough and user friendly enough to be used in smaller laboratories ${ }^{14-16}$.

An equivalent background subtraction method to that presented here using off the shelf components uses a boxcar averager feeding into a lock - in amplifier ${ }^{9}$. This also results in a demodulated signal stream, with a point added at the end of each laser modulation cycle. Zero dead time boxcars are now commercially available with performance matching or exceeding what we present here. Faster speeds may also be obtained with fast digitizing oscilloscopes, but these do not do the demodulation that we present here. In either case, synchronizing this process over many PMTs is difficult as a large number of physical devices are required, and any mis- 
alignment in synchronization is twofold damaging to the result.

What we seek, then, is to introduce a different strategy for acquiring modulated LIF data across multiple arrays of PMTs. This strategy is made possible by FPGAs. A single chip can acquire data that would take 32 sets of boxcar / lock - in combinations. Moreover, the data acquired the way we present is ready to be processed on the FPGA, a possibility that is missing from a boxcar - lock in combination. This is particularly useful in applications that require statistical averaging, since a running average will both reduce signal to noise ratio and constrain the needed storage space to a constant.

In this Article, we present an FPGA based up / down counter LIF demodulation scheme. We show how to synchronize the asynchronous digitized pulses to the FPGA clock domain and the implementation of the up / down counters for multiple parallel channels. This scheme demodulates the LIF data on a single device, resulting in a digitized stream of LIF photon counts. This stream is ready for time domain analysis, such as detecting plasma fluctuations through cross correlations. We verify the FPGA based LIF demodulation on 32 data channels from two 16 - element PMTs by comparing it to a traditional lock-in LIF measurement. Finally, we show an example of an application of this method by using the digitized LIF photon stream to calculate a cross correlation and statistically average it with time. This averaged cross correlation is used to calculate a spectrum of a plasma.

This FPGA based demodulation scheme is important for any type of LIF measurement that requires a digitized stream of background subtracted LIF photons, since it removes a major component of measurement deadtime. It is particularly useful in a measurement where statistical averaging is required to achieve a good signal to noise ratio.

\section{SYSTEM DESIGN}

The system is housed in a Versa Module Europa bus (VMEbus) crate, a CAEN SpA VME8002. VMEbus is a bus architecture that provides both housing for electronics circuit boards and a communications interface connecting the boards to each other through memory addressing. The pulse trains from two separate 16 element PMTs, Hamamatsu model H10515B-200, are fed into two 16 element falling edge discriminators, CAEN SpA model V895, via coaxial cables with LEMO connectors. The 16 element PMTs may be used for increased spatial resolution, but here we simply sum them after the FPGA based demodulation to improve our signal to noise ratio. The discriminators convert the PMT data pulses into ECL logic pulses. The resulting ECL logic pulse train is sent via ribbon cable into a VME board, CAEN Spa model V2495, housing a user programmable FPGA (UFPGA), an Altera Cyclone V 5CGXBC4C6F27C7. On the UFPGA, the data is demodulated, stored in on - UFPGA memory, and sent to the computer through the VMEbus. This high level system design is shown in Fig. 1. A Struck GmbH SIS3153 provides a USB interface to the

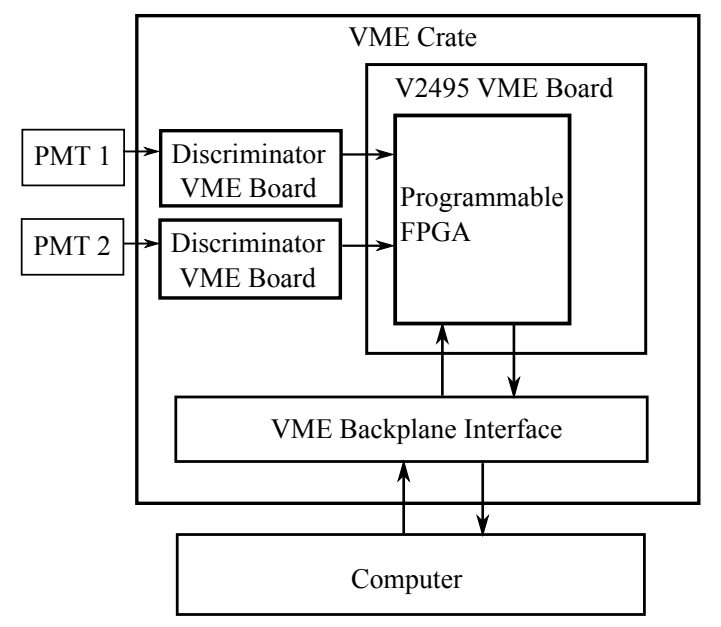

FIG. 1. The high level system design. This shows the individual boards used in the VME data acquisition system, and their connections to the PMTs and the computer.

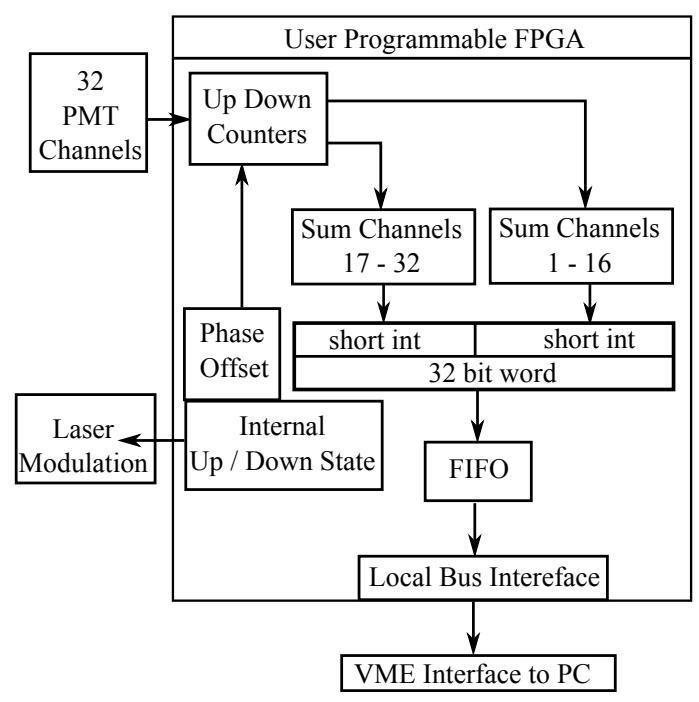

FIG. 2. Logic on the user programmable FPGA. This logic handles three major tasks: input / output, the up / down counters, and storage of the demodulated LIF counts before it is read to the computer (FIFO).

computer to connect to the VMEbus.

The logic on the UFPGA is laid out in Fig. 2. The 32 channel ECL logic pulses, 16 from each PMT / discriminator pair, are fed into 32 up / down counter channels on the UFPGA. A periodic internal up / down state, created by a clock synchronized internal counter, governs whether the counters count up or down. This signal is also fanned out with a user configurable phase to a TTL output connector, providing a control signal for laser modulation. This signal's - and thus the laser modulation's - frequency is either $1 \mathrm{MHz}$ or $100 \mathrm{kHz}$. At each rising edge of this laser state the counters' values are sampled and then reset to zero. Each sample from the 16 counters is summed and the result drives an $\mathrm{Al}-$ tera FIFO input line ${ }^{17}$. The FIFO output is connected to a local bus interface, which interfaces with the VME backplane via the V2495 hardware and is read out by the PC. 
A finite state machine, not shown in Fig. 2, controls the status of the UFPGA, whether it is acquiring data, reading out the FIFO to the $\mathrm{PC}$ via VME, or idling. The user changes the FSM state through adjusting control register bits via VME memory write accesses.

\section{UP DOWN COUNTER DESIGN}

The up / down counter requires a few intricacies. Firstly, the input pulses are ECL logic pulses of width 5 ns while the UFPGA clock is $50 \mathrm{MHz}$. Secondly, these input pulses are not synchronized. Thus the problem is that one needs to bring asynchronous $200 \mathrm{MHz}$ clock signals into a $50 \mathrm{MHz}$ clock domain. This is solved using a Flancter ${ }^{18}$ circuit that has been modified to count up or down based on an input status signal ${ }^{19}$. The Flancter uses a twisted pair of flip flops to set a status flag when an incoming pulse is detected. The pulse needs to be long enough to trip a flip flop - generally only $1 \mathrm{~ns}$.

The flancter circuit is controlled by a finite state machine that requires four clock cycles after a data pulse is latched to increment a counter. Pulses that arrive during this state would be missed. This is another setback in the process of counting these faster data pulses.

This problem is solved by taking advantage of the massively parallel nature of FPGAs. Each up / down counter has a configurable (at compile time) number of flancter circuits. Busy signals from occupied flancters are input to an addresser in order to route the next pulse to an available flancter. Since the user can scale the number of flancters as needed, the bandwidth of the counting circuit is limited by FPGA signal propagation times. This design is robust with respect to varying input data pulse lengths. Fig. 3 shows a diagram of the flancter busy line addresser and demutiplexer on the input PMT pulse train.

\section{EXPERIMENT SETUP}

The UFPGA is tested on a laboratory plasma device set up to obtain laser induced fluorescence, shown in Fig. 4. The plasma is a cylidrical axially magnetized plasma column of singly ionized Argon (Ar II) created with an RF inductively coupled source. Ion density and electron temperature are measured with a Langmuir probe and are typically $n_{i} \approx 5 e 9 \mathrm{~cm}^{-3}$ and $T_{e} \approx 8$ $\mathrm{eV}$. LIF traditionally applied shows the ion temperature $T_{i} \approx 0.08 \mathrm{eV}$. The plasma column is $230 \mathrm{~cm}$ long with radius $\approx 2.5 \mathrm{~cm}$. Current carrying coils ensconce the chamber in a solenoid to create an axial magnetic field of 667 Gauss. The plasma chamber also contains two independently movable sets of light collecting optics focused on the axis of the plasma.

These light collection optics gather light from the plasma which includes laser induced fluorescence. They both spatially filter via a pinhole and spectrally filter via optical filters centered near $442 \mathrm{~nm}$. The laser power at the plasma chamber point of entry is $30 \mathrm{~mW}$ - which is already close to saturation broadening for our plasma.
The laser is a TOPTICA TA 100 Diode laser. Laser amplitude modulation is achieved with a Conoptics electro-optic modulator model 390-2P. This laser excites the Ar II metastable state $3 \mathrm{~s}^{2} 3 \mathrm{p}^{4}\left({ }^{3} \mathrm{P}\right) 3 \mathrm{~d}{ }^{4} \mathrm{~F}_{7 / 2}$ with 668 nm light to the state $3 \mathrm{~s}^{2} 3 \mathrm{p}^{4}\left({ }^{3} \mathrm{P}\right) 4 \mathrm{p}^{4} \mathrm{D}_{5 / 2}^{\circ}$, which then decays to $3 \mathrm{~s}^{2} 3 \mathrm{p}^{4}\left({ }^{3} \mathrm{P}\right) 4 \mathrm{p}{ }^{4} \mathrm{P}_{3 / 2}^{\circ}$ while emitting light near 442 $\mathrm{nm}^{20}$. The laser wavelength is tunable across the Zeeman split absorption spectrum of the ${ }^{4} \mathrm{~F}_{7 / 2} \rightarrow{ }^{4} \mathrm{D}_{5 / 2}^{\circ}$ transition. By tuning the laser wavelength, we can measure the transition absorption spectrum that has been broadened by the ion velocity distribution function of the plasma.

\section{LOCK-IN VERIFICATION}

For verification, a lock-in is also connected to the discriminators. The V895 discriminators, in addition to outputting ECL logic pulses, also output a single voltage signal proportional to the number of currently activated discriminators. This signal is sent through an analog RC low pass filter with $\mathrm{RC} \approx 1 \mu$ s then into a Zurich Instruments MFLI5M lock-in amplifier. The lock-in reference frequency is set to the laser modulation frequency.

Two sets of data are taken to verify the system. The first we take by scanning the laser across the entire absorption spectrum while reading the output from the lock-in. The lock-in internal RC time constant is slow relative to the laser scanning speed. The second data set is taken with the UFPGA. At a given wavelength, $6.4 \mathrm{~s}$ of up / down data are taken and then averaged to give an LIF rate. This data set length was chosen arbitrarily to ensure a high signal to noise ratio. This is repeated for wavelengths across the entire absorption spectrum. The results are shown in Fig. 5.

This measurement, while useful for verification since it recreates the output of a lock-in amplifier, is time consuming and is not the strength of the UFPGA setup. Indeed, when used this way, the UFPGA is slower and has worse resolution than the lock-in. The UFPGA setup's strength is an application that needs a digitized stream (or streams), in time, of LIF photon counts. A lock-in amplifier is not useful for obtaining this type of data. We show a cross correlation as an example of this kind of application.

\section{EXAMPLE APPLICATION: CROSS CORRELATION}

Cross correlation is a powerful tool for isolating and removing photon statistics noise. As an example, we provide a program which takes the demodulated time series data from each of the two sets of counters, cross correlates it, and takes a running pointwise average of the cross correlation with earlier cross correlations. By doing this, we have created a process that finds the spectrum of plasma fluctuations by reducing the photon statistics noise floor through time averaging.

We choose cross correlation as an example because it demonstrates the strengths of this FPGA based demodulation. There is no processing needed for demodulation. The digitized LIF stream is directly used to calculate the 


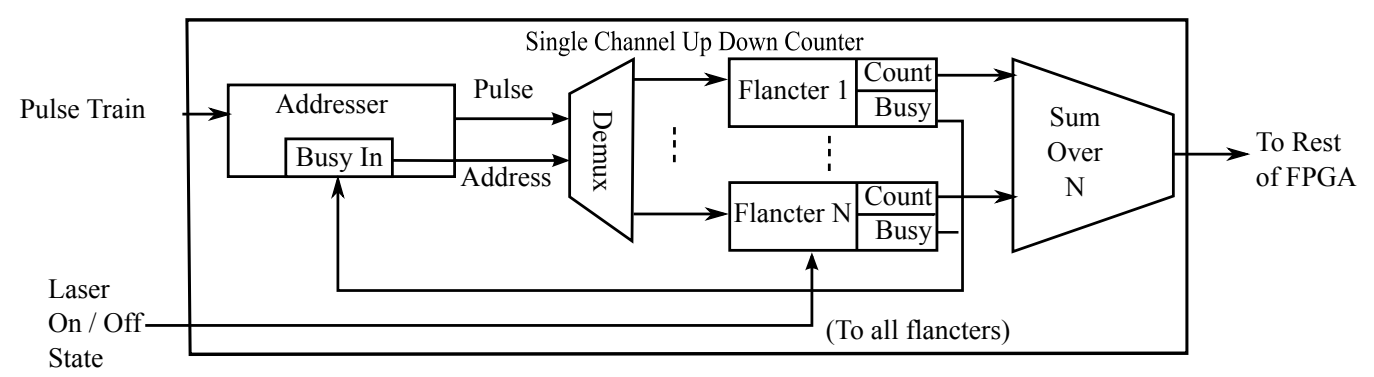

FIG. 3. Block design of a single channel's up / down counter. Each incoming pulse is automatically routed to an idle flancter so that no pulse is missed.

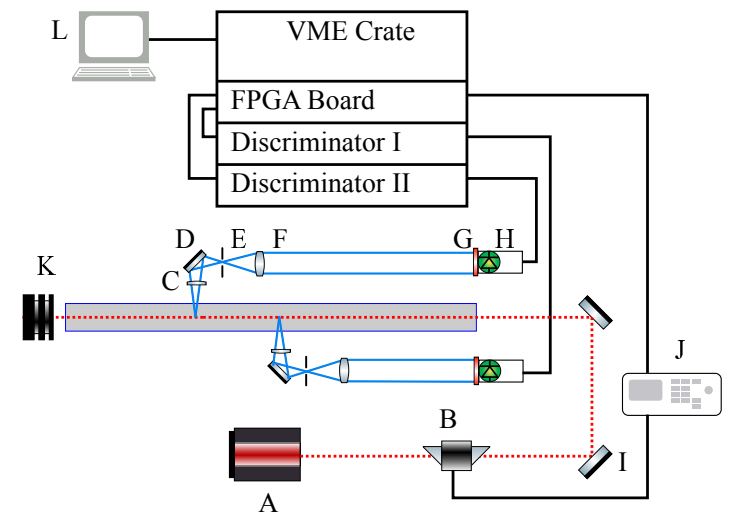

FIG. 4. Experimental set up for acquiring LIF to test the FPGA. A - diode laser. B - electro optical modulator. C - aspheric lens. D - mirror. E - pinhole. F - collimating Fresnel lens. G - $442 \mathrm{~nm}$ filter (1nm bandwidth) H - Hamamatsu H10515B-200 PMT. I - mirrors. J - arbitrary function generator. K - beam dump. L - data acquisition PC. This setup can acquire streams of photons from two independent and moveable positions.

cross correlation. Successive cross correlations can be statistically averaged to improve the signal to noise ratio. An application such as this that requires statistical averaging is where the digitized data from this FPGA is advantageous compared to a lock-in.

We accomplish this with a $\mathrm{C}$ program on the data acquisition computer. The program first sets up the UFPGA with the desired parameters for the experiment. It then lets the UFPGA acquire until it fills the onboard FIFO - 32k elements. Then it downloads the time series demodulated LIF data through the VME backplane. With this LIF data set, it performs a cross correlation and adds the result to a running average. Cross correlation is done in frequency space with the assistance of the FFTW3 libraries ${ }^{21}$. With this optimization it takes less time to perform the cross correlation than to acquire data. To take advantage of this, the acquisition loop is split into two threads using the OpenMP library ${ }^{22}$. Thread blocks are in place to ensure that, when one thread is acquiring data, the other is calculating a cross correlation of its already acquired data set. With this scheme, the only data dead time is during data acquisition itself and transferring data to the computer.

One minute of demodulated LIF data, in 32ms groups with a $1 \mathrm{MHz}$ laser modulation frequency, was acquired

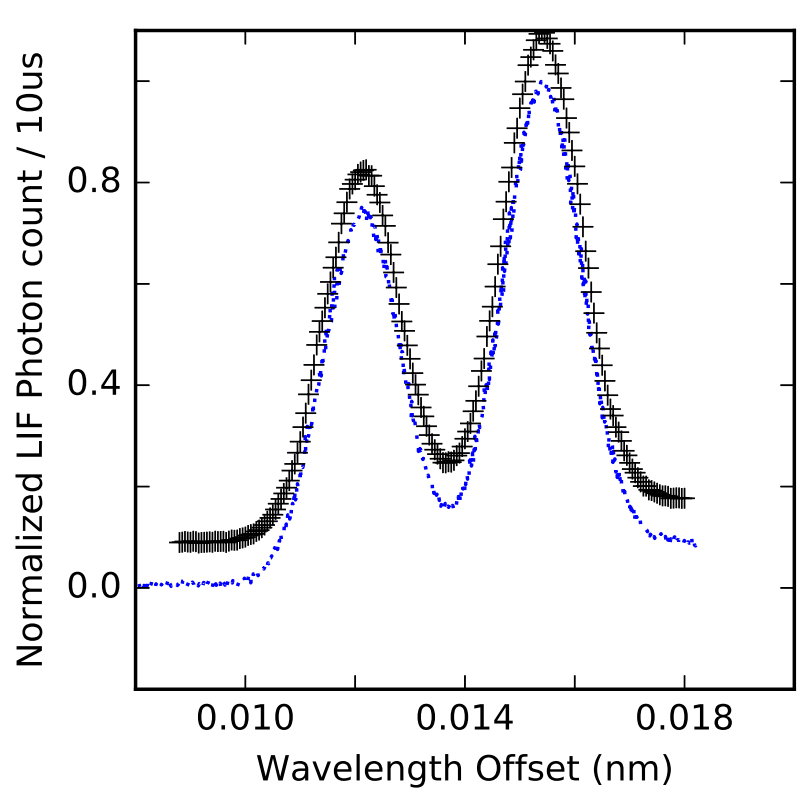

FIG. 5. Lock-in data and FPGA absorption spectra. The dotted line denotes lock-in data and the plus $(+)$ symbols correspond to FPGA data. The abscissa is the positive offset from $668.6 \mathrm{~nm}$. The two data sets have been normalized relative to each dataset's peak. Note the zero of the FPGA data has been positively offset to make comparison easier. Zeeman splitting of the transition is apparent in these curves.

and averaged with this setup. $32 \mathrm{~ms}$ groups were taken since this is how long the $32 \mathrm{~K}$ element FIFO on the UFPGA fills up at $1 \mathrm{MHz}$. An upper bound time to transfer this data from the UFPGA to the computer is $10 \mathrm{~ms}$. To see the fluctuation spectrum, the final statistically averaged cross correlation function is point wise multiplied with a Gaussian windowing function of width $5 \mathrm{~ms}$ and Fourier transformed. The resulting spectrum is shown in Fig. 6. A standing wave bounded mode in the plasma chamber from the ion acoustic mode is visible at $1.2 \mathrm{kHz}$ and a drift wave peak is visible near $f^{*} \approx 10 \mathrm{kHz}$.

A traditional VME histogramming scalar acquisition obtains a similar spectrum from about 20 minutes of acquisition, writing about 5 gigabytes of raw data that then must be demodulated, cross correlated, and statistically averaged afterwards. Moreover, the laser modulation frequency is only $100 \mathrm{kHz}$. In comparison, the FPGA and $\mathrm{C}$ program we present writes a $500 \mathrm{kB}$ file containing 


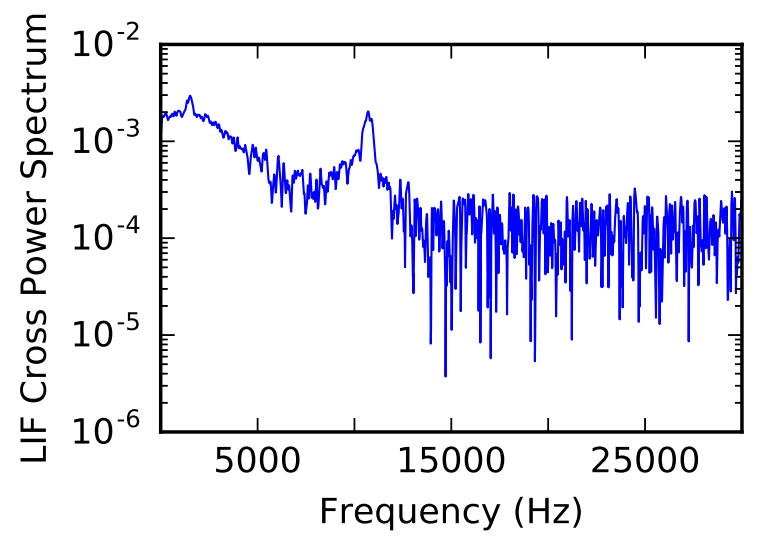

FIG. 6. Gaussian windowed spectrum of the time averaged cross correlation of the demodulated LIF data streams. An ion acoustic peak at $1.2 \mathrm{kHz}$ and a drift peak near $10 \mathrm{kHz}$ are visible. Above this the plasma exhibits noise.

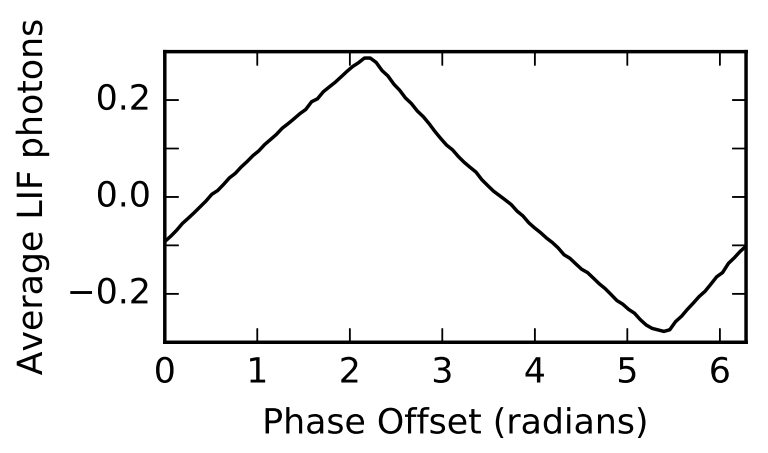

FIG. 7. Average LIF signal per up / down counting cycle when the laser logic signal is phase shifted. The offset giving a maximum is chosen for a given setup. This can be adjusted when the setup changes. A $1 \mathrm{MHz}$ laser modulation frequency was used here. The triangle wave verifies the up / down counters are counting LIF photons.

60 seconds worth of statistically averaged cross correlation after 1.5 minutes with a $1 \mathrm{MHz}$ laser modulation frequency. While the dead time is not eliminated, it has been drastically reduced. With our particular set up, the FPGA makes possible both the demodulation, and thus dead time reduction, and the faster laser modulation frequency.

While this particular plasma only has noise at higher frequencies, we have validated the FPGA's ability to find fluctuation spectra for higher sampling frequency. This, combined with the removal of the dead time needed to demodulate the LIF signal, means that this FPGA demodulation method is useful for finding higher frequency plasma fluctuation spectra for low signal to noise ratio plasmas.

\section{SUMMARY AND FUTURE WORK}

We presented a user programmable FPGA data acquisition system that gives a stream of LIF photon counts that is updated as laser amplitude modulation cycle completes. This system was verified against a lock-in measurement of the absorption spectrum of a Zeeman split transition line in a singly charged Argon ion magnetized plasma. We also presented a $\mathrm{PC}$ based $\mathrm{C}$ program for finding the cross correlation of the demodulated LIF data. Averaging this cross correlation over time lowers the photon statistics noise floor, making more detailed nonperturbative measurements of the ion density or temperature fluctuation spectrum through LIF possible.

Future work should take greater advantage of the strengths of an FPGA. This includes moving the cross correlation, or another application that requires time averaging, to the user programmable FPGA itself so that it removes the UFPGA to PC data transfer bottleneck. Another possibility is integrating impulse response filters in the FPGA logic, combining the strengths of a lock-in amplifier with the strengths of a digitized stream of LIF photons. It is possible that the dead time can be completely eliminated for these applications, leaving only the time required to achieve a desirable signal to noise ratio.

Finally, the FPGA makes spectral measurements possible at much higher frequencies. A science avenue of future work involves observing high frequency fluctuations with LIF closer to this new Nyquist frequency of 500 $\mathrm{kHz}$. It is also possible, by doubling the FPGA based phased-lock-loop output clock frequency, to increase the laser modulation frequency to $2 \mathrm{MHz}$.

\section{SUPPLEMENTARY MATERIAL}

The VHDL code for the up / down counters, the C code for the cross correlation, and supporting files for the V2495 board are provided as supplementary material for this paper. Both a Quartus archive (.qar) of the entire project and a zip file of only the VHDL files is included. The code for finding the correct laser logic signal offset by scanning the relative phase through all values is also included. A typical phase scan is shown in Fig. 7. This serves as another verification of the expected behavior of the up down counters. The entire FPGA project was written in and compiled using Quartus II 15.0 64 bit Web Edition, which is free, while the $\mathrm{C}$ code was written in Eclipse.

\section{ACKNOWLEDGEMENTS}

The first author wishes to acknowledge a donation of useful lab equipment from Ingsheng Kung and the Kung family. This work is supported by the US DOE under the NSF-DOE program with grant number DE-FG0299ER54543.

\footnotetext{
${ }^{1}$ R. A. Stern and J. A. Johnson, Phys. Rev. Lett. 34, 1548 (1975).

${ }^{2}$ A. V. Gorbunov, N. A. Molodtsov, I. V. Moskalenko, and D. A. Shcheglov, Rev. Sci. Instrum. 81 (2010), http://dx.doi.org/10.1063/1.3475799.

${ }^{3}$ N. Hershkowitz and R. A. Breun, Rev. Sci. Instrum. 68, 880 (1997).

${ }^{4}$ T. S. Strickler, T. K. Langin, P. McQuillen, J. Daligault, and T. C. Killian, Phys. Rev. X 021021, 1 (2016), arXiv:1512.02288.
} 
${ }^{5}$ F. Anderegg, X.-P. Huang, E. Sarid, and C. F. Driscoll, Rev. Sci. Instrum. 68, 2367 (1997).

${ }^{6}$ R. A. Stern, Rev. Sci. Instrum. 56, 1006 (1985).

${ }^{7}$ F. Skiff and F. Anderegg, Phys. Rev. Lett. 59, 896 (1987).

${ }^{8}$ A. Diallo and F. Skiff, Phys. Plasmas 12, 110701 (2005).

${ }^{9}$ M. J. Rutledge, M. E. Tremblay, and J. D. Winefordner, Appl. Spectrosc. 41, 5 (1987).

${ }^{10}$ B. Pelissier and N. Sadeghi, Rev. Sci. Instrum. 67 (1996), 10.1063/1.1136741.

${ }^{11}$ G. Naylor, in Rev. Sci. Instrum., Vol. 81 (2010) p. $10 \mathrm{E} 110$.

12 J. Lovell, G. Naylor, A. Field, P. Drewelow, and R. Sharples, in Rev. Sci. Instrum., Vol. 87 (2016) p. 11E721.

${ }^{13}$ I. Balboa, B. Huang, G. Naylor, M. Walsh, A. Sirinelli, P. Parsons, J. Fessey, M. Townsend, M. Beurskens, N. Conway, J. Flanagan, M. Kempenaars, and A. Kirk, in Rev. Sci. Instrum., Vol. 81 (American Institute of Physics, 2010) p. 10D534.

${ }^{14}$ A. Schwettmann, J. Sedlacek, and J. P. Shaffer, Rev. Sci. Instrum. 82, 103103 (2011).
${ }^{15}$ A. Ryou and J. Simon, Rev. Sci. Instrum. 88, 013101 (2017), arXiv:1604.04668.

${ }^{16}$ N. Lusardi, J. W. N. Los, R. B. M. Gourgues, G. Bulgarini, and A. Geraci, Rev. Sci. Instrum. 88, 035003 (2017).

17 "Altera SCFIFO and DCFIFO IP Cores User Guide," (2017), http://www.altera.com.

${ }^{18}$ R. Weinstein, Xilinx Xcell J. 37, 54 (2000).

${ }^{19} \mathrm{~A}$ good explanation of a similar circuit using a Flancter can be found at https://www.doulos.com/knowhow/fpga/ fastcounter/.

${ }^{20}$ A. Kramida, Y. Ralchenko, J. Reader, and N. A. S. D. Team, "NIST Atomic Spectra Database (5.2)," (2014).

${ }^{21}$ M. Frigo, ACM SIGPLAN Not. 34, 169 (1999).

22 "OPENMP Application Program Interface Version 3.1," (2011), http://www . openmp.org/wp-content/uploads/OpenMP3.1.pdf. 


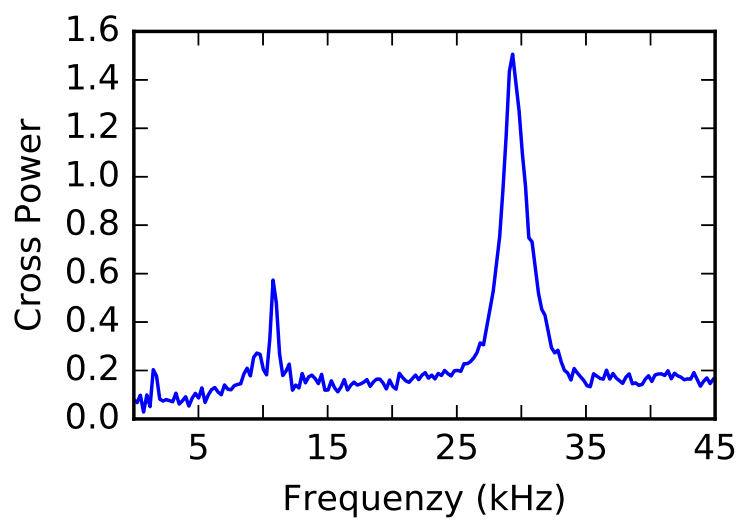

